\title{
SAMPLING THEOREMS FOR NONSTATIONARY RANDOM PROCESSES
}

\author{
BY
}

ALAN J. LEE

\begin{abstract}
Consider a second order stochastic process $\{X(t), t \in \mathbf{R}\}$, and let $H(X)$ be the Hilbert space generated by the random variables of the process. The process is said to be linearly determined by its samples $\{X(n h), n \in \mathbf{Z}\}$ if the random variables $X(n h)$ generate $H(X)$. In this paper we give a sufficient condition for a wide class of nonstationary processes to be determined by their samples, and present sampling theorems for such processes. We also consider similar problems for harmonizable processes indexed by LCA groups having suitable subgroups.
\end{abstract}

1. Introduction. It is well known that a second order, zero mean, weakly stationary random process $\{X(t), t \in \mathbf{R}\}$ satisfies the sampling expansion

$$
X(t)=\sum_{n=-\infty}^{\infty} X(n h) \frac{\operatorname{Sin} \pi h^{-1}(t-n h)}{\pi h^{-1}(t-n h)}
$$

if the spectral measure $\mu$ of $X(t)$ is supported by the interval $\left(-h^{-1} / 2, h^{-1} / 2\right)$. This so called "sampling theorem" dates back to Cauchy and is of considerable importance in communication theory; such processes with bounded spectra are called "band-limited".

This concept of "band-limitedness" can easily be generalized to nonstationary processes; see e.g. Zakai [1], Piranashvili [2], Lee [3], [4]. A second order random process, not necessarily stationary, is said to be "band-limited to $w$ " if its covariance function $R(t, s)=E(X(t) \overline{X(s)})$ has a Fourier transform $\hat{R}$ (possibly a distribution) supported by the square $[-w, w] \times[-w, w]$ in $\mathbf{R}_{2}$. If $h^{-1} / 2>w$, then a band-limited process satisfies a modified sampling theorem similar to (1). These sampling theorems, which converge in mean square and also almost surely, enable a band-limited process to be exactly reconstructed from its samples $\{X(n h), n \in \mathbf{Z}\}$. Of course, a process need not be band-limited to admit an error-free reconstruction from its samples. Lloyd [5] gave a necessary and sufficient condition on the spectral measure for a stationary process to admit such a reconstruction. More

Received by the editors March 25, 1976 and, in revised form, March 28, 1977.

AMS (MOS) subject classifications (1970). Primary 60G99, 94A05; Secondary 42A68, 43A25, 46E35.

Key words and phrases. Sampling theorem, reproducing kernel Hilbert space, Sobolev space, Fourier series, periodic distribution, harmonizable process. 
precisely, let $L_{2}(\Omega)$ be a Hilbert space of square integrable random variables on some probability space $(\Omega, Q, P)$, and let $H(X)$ be the closed subspace of $L_{2}(\Omega)$ generated by the random variables $\{X(t): t \in \mathbf{R}\}$ of the random process $X(t)$. The process can in principle be exactly reconstructed from its samples $X(n h)$ if $H(X)=\mathscr{N}$ where $\Re$ is the closed subspace of $L_{2}(\Omega)$ generated by $\{X(n h): n \in \mathbf{Z}\}$. Lloyd uses the terminology " $x$ is linearly determined by its samples" in this case. He proved that this will be the case if and only if the spectral measure $\mu$ of $X(t)$ has a support $\Lambda$ such that the translates of $\Lambda$ by $n h^{-1}$ are disjoint for every integer $n$. Rao [6] extended Lloyd's result to the case of harmonizable processes, but Rao's condition is not necessary, as is shown in $\$ 4$.

If a process $X(t)$ is linearly determined by its samples, then it is possible to develop sampling expansions for $X(t)$. Lloyd gives such an expansion which converges in mean square and also almost surely in the case when $X(t)$ is stationary.

In this paper we consider a wide class of non-band-limited processes and give sufficient conditions similar to Lloyd's for a process to be "determined by its samples". We also give explicit sampling expansions which permit error-free interpolation, in the spirit of those given by Lloyd in the stationary case. These sampling expansions are shown to converge in mean square and also almost surely.

First, processes whose covariance functions are square integrable with respect to Lebesgue measure on the plane are considered, and then the results obtained are extended to processes whose covariance functions satisfy a more general integrability condition, namely covariances square integrable with respect to the measure $\left(1+t^{2}+s^{2}\right)^{-k} d t d s$ for some integer $k$. We then briefly sketch a counterexample to a theorem of Rao [6] concerning the sampling of harmonizable random processes. Finally, sampling results are presented for harmonizable processes indexed not by $\mathbf{R}$ but by arbitrary locally compact abelian topological groups containing certain types of subgroups.

In the sequel we will make use of certain results from the theory of distributions. As usual, the space of $C^{\infty}$ functions with compact support is denoted by $\mathscr{D}$ (the space of test functions) and the Schwartz space of rapidly decreasing functions by $\mathcal{S}$. We also make use of the Sobolev spaces $H^{2, k}\left(\mathbf{R}_{n}\right)$, where for $k>0, H^{2, k}\left(\mathbf{R}_{n}\right)$ consists of all distributions $u$ on $\mathbf{R}_{n}$ such that all the derivatives of order $<k$ of $u$ belong to $L_{2}\left(\mathbf{R}_{n}\right)$. For $k<0, H^{2, k}\left(\mathbf{R}_{n}\right)$ consists of all distributions on $\mathbf{R}_{n}$ that are the finite sum of derivatives of order $\leqslant k$ of functions in $L_{2}\left(\mathbf{R}_{n}\right)$. Every distribution in $H^{2, k}\left(\mathbf{R}_{n}\right)$ is the Fourier transform of a function on $\mathbf{R}_{n}$ square integrable with respect to the measure $\left(1+|t|^{2}\right)^{k} d t$, and for $k>0$, every element $u$ of $H^{2,-k}\left(\mathbf{R}_{n}\right)$ has the 
canonical representation

$$
u=\sum_{|i|<k}(-1)^{|i|}\left(\frac{\partial}{\partial x}\right)^{2 i} f
$$

where $i=\left(i_{1}, \ldots, i_{n}\right),|i|=i_{1}+\cdots+i_{n}$,

$$
\left(\frac{\partial}{\partial x}\right)^{2 i}=\frac{\partial^{2 i_{1}} \cdots \partial^{2 i_{n}}}{\partial x_{1}^{2 i_{1}} \cdots \partial x_{n}^{2 i_{n}}}
$$

for nonnegative integers $i_{1} \cdots i_{n}$ and $f$ is a function in $H^{2, k}\left(\mathbf{R}_{n}\right)$ that is the limit of a sequence of testfunctions in $H^{2, k}\left(\mathbf{R}_{n}\right)$.

We also make use of the following series representation of a measurable, mean square continuous zero mean second order stochastic process $\{X(t), t$ $\in \mathbf{R}\}$ due to Cambanis and Masry [7]. Suppose that the covariance $R(t, s)$ satisfies $\int R(t, t) \mu(d t)<\infty$ for some measure $\mu$ equivalent to Lebesgue measure. Then we may define an operator $R$ on $L_{2}(R, \mu)$, the space of functions square integrable with respect to $\mu$, by $R f(t)=\int R(t, s) f(s) \mu(d s)$. $R$ is a bounded trace class operator, with nonzero eigenvalues $\lambda_{1}, \lambda_{2}, \ldots$ corresponding to eigenfunctions $f_{1}, f_{2}, \ldots$ Then there exists an orthogonal basis $e_{1}, e_{2}, \ldots$ for $H(X)$ such that $X(t)=\sum_{j=1}^{\infty} f_{j}(t) e_{j}$ and $R(t, s)=\Sigma \lambda_{j} f_{j}(t) \overline{f_{j}(s)}$.

Finally, we record the notation employed in $\S 4$. We denote a LCA group by $G$, and the character group of $G$ (the group of continuous homomorphisms from $G$ to the unit circle) by $\hat{G}$. If $\alpha \in \hat{G}$, we write the value of $\alpha$ at $g \in G$ as $\langle\alpha, g\rangle$. The collection of complex, regular Borel measures of finite variation on $G$ is written $M(G)$; the support of such a measure $\mu \in M(G)$ is the set of all points $g \in G$ such that for every open neighborhood $U$ of $g$, there exists a Borel set $E$ with $\mu(E) \neq 0$ and $E \subseteq U$.

2. Sampling theorems for processes with square integable covariances. In the sequel, we assume the process $\{X(t), t \in \mathbf{R}\}$ to be a measurable second order, zero mean process with continuous covariance function $R(t, s)=$ $E(X(t) \overline{X(s)})$. In this section we assume in addition that $\int R(t, t) d t<\infty$. (In what follows all integrals are to be taken over the whole real line unless otherwise specified.) Under these conditions $X(t)$ has a series expansion (converging in mean square)

$$
X(t)=\sum_{j=1}^{\infty} f_{j}(t) e_{j}
$$

where the "time functions" $f_{j}$ are the eigenfunctions of the operator $R$ on $L_{2}(\mathbf{R})$ with kernel $R(t, s)$ corresponding to nonzero eigenvalues $\lambda_{j}$, and the r.v.'s $e_{j}$ form an orthogonal basis for $H(X)$ with $E\left(e_{i} \bar{e}_{j}\right)=\lambda_{j} \delta_{i j}$. Moreover, the covariance function $R$ has the representation 


$$
R(t, s)=\sum_{j=1}^{\infty} \lambda_{j} f_{j}(t) \overline{f_{j}(s)}
$$

the convergence being absolute. (Cambanis and Masry [7, Theorem 6].) Let $\nu$ be a measure which assigns mass $\lambda_{j}$ to the point $j$. Since $R$ is trace-class as an operator $L_{2}(\mathbf{R}) \rightarrow L_{2}(\mathbf{R}), \nu$ is a finite positive measure and $R(t, s)$ can be written

$$
R(t, s)=\int f(t, \lambda) \overline{f(s, \lambda)} \nu(d \lambda)
$$

where $f(t, \lambda)=f_{j}(t)$ for $\lambda=j$. It follows that the space of all functions of the form

$$
g(\cdot)=\sum_{j=1}^{\infty} \lambda_{j} f_{j}(\cdot) \overline{g_{j}}
$$

where $\left\{g_{j}\right\}$ is a sequence of complex numbers satisfying $\sum_{j=1}^{\infty} \lambda_{j}\left|g_{j}\right|^{2}<\infty$, is the reproducing kernel Hilbert space (RKHS) $H(R)$ with inner product $(g, h)=\Sigma \lambda_{j} g_{j} \bar{h}_{j}$ which represents the process $X(t)$ in the sense of Parzen [8]. The spaces $H(X)$ and $H(R)$ are isomorphic, with $X(t)$ corresponding to the function $R(\cdot, t)=\sum_{j=1}^{\infty} \lambda_{j} f_{j}(\cdot) \overline{f_{j}(t)}$ in $H(R)$. It follows that $H(X)=\mathfrak{N}$ if and only if the closed subspace $\Re_{R}$ generated by the set of functions of the form $\sum_{j=1}^{\infty} \lambda_{j} f_{j}(\cdot) \overline{f_{j}(n h)}, n \in \mathbf{Z}$, equals $H(R)$. This will be the case if and only if for any $g \in H(R)$ with $g \perp \mathfrak{T}_{R}, g=0$.

THEOREM 1. Let $\hat{R}$ be the $L_{2}(\mathbf{R} \times \mathbf{R})$ Fourier transform of $R(t, s)$. Let $\Lambda$ be the support of $R$ considered as a distribution in the plane. If the translates $\Lambda+\left(n h^{-1}, n h^{-1}\right)$ of $\Lambda$ are disjoint for every $n$, then $H(R)=\Re_{R}$ and $H(x)=\Re$.

Proof. The series (3) converges in $L_{2}(\mathbf{R} \times \mathbf{R})$ so we may take Fourier transforms of both sides to obtain

$$
\hat{R}(x, y)=\sum_{j=1}^{\infty} \lambda_{j} \hat{f}_{j}(x) \overline{\hat{f}_{j}(y)} .
$$

Now let $\phi \in \mathscr{D}\left(\mathbf{R}_{1}\right)$, the space of $C^{\infty}$ functions on the line with compact support, and write $\hat{R}, \hat{f_{j}}$ to denote the distributions on $\underline{\mathbf{R}}_{2}$ and $\mathbf{R}$ corresponding to the locally integrable functions $\hat{R}, \hat{f}_{j}$. Then if $\phi \otimes \bar{\phi}(x, y)=\phi(x) \overline{\phi(y)}$,

$$
\begin{aligned}
\hat{R}(\phi \otimes \bar{\phi}) & =\iint \sum_{j=1}^{\infty} \lambda_{j} \hat{f}_{j}(x) \overline{\hat{f}_{j}(y)} \phi(x) \overline{\phi(y)} d x d y \\
& =\sum_{j=1}^{\infty} \lambda_{j} \iint \hat{f}_{j}(x) \overline{\hat{f}_{j}(y)} \phi(x) \overline{\phi(y)} d x d y \\
& =\sum_{j=1}^{\infty} \lambda_{j}\left|\hat{f}_{j}(\phi)\right|^{2} \geqslant \lambda_{j}\left|\hat{f}_{j}(\phi)\right|^{2} \quad \text { for all } j .
\end{aligned}
$$


Now let $\Lambda_{j}$ be the support of $\hat{f}_{j}$, and let $\Lambda_{0}=\{x:(x, x) \in \Lambda\}$. We will show that $\Lambda_{j} \subseteq \Lambda_{0}$ for each $j$ and that the translates of $\Lambda_{0}$ by $n h^{-1}$ are all disjoint. Let $x \in \Lambda_{j}$, and let $U$ be a neighbourhood of $(x, x)$ in $\mathbf{R}_{2}$. Let $V$ be a neighbourhood of $x$ in $\mathbf{R}_{1}$ with $V \times V \subseteq U$. Then there exists a $\phi \in \mathbb{D}$ supported by $V$ with $f_{j}(\phi) \neq 0$, so $\phi \otimes \bar{\phi}$ is in $\mathscr{D}\left(\mathbf{R}_{2}\right)$, is supported by $U$ and $\hat{R}(\phi \otimes \bar{\phi}) \geqslant \lambda_{j}\left|f_{j}(\phi)\right|^{2} \neq 0$, so $\hat{R}(\phi \otimes \bar{\phi}) \neq 0$ and hence $(x, x) \in \Lambda$. Thus $x \in \Lambda_{0}$. Suppose that $n h^{-1}+\Lambda_{0}, m h^{-1}+\Lambda_{0}$ are translates of $\Lambda_{0}$ with nonempty intersection containing a point $x$ say. Then $x-n h^{-1} \in \Lambda_{0}$, so $(x, x) \in\left(n h^{-1}, n h^{-1}\right)+\Lambda$. Similarly $(x, x) \in\left(m h^{-1}, m h^{-1}\right)+\Lambda$ contradicting the hypothesis that the translates of $\Lambda$ are disjoint. Now let $g \in H(R)$, $g \perp \Re_{R}$. Set $g(\cdot)=\sum_{j=1}^{\infty} \lambda_{j} f_{j}(\cdot) \bar{g}_{j}$, then $g \in L_{2}\left(\mathbf{R}_{1}\right)$ and $g(n h)=0$ for all $n \in \mathbf{Z}$. Taking Fourier transforms, $\hat{g}(x)=\sum_{j=1}^{\infty} \lambda_{j} \hat{f}_{j}(x) \bar{g}_{j}$, so supp $\hat{g} \subseteq \Lambda_{0}$. Now let $\hat{g}_{n}(x)=\hat{g}\left(x-n h^{-1}\right)$; then the functions $\hat{g}_{n}$ are orthogonal in $L_{2}\left(\mathbf{R}_{1}\right)$ since their supports are disjoint. Let $\phi$ be a function in the Schwartz space $\mathcal{S}$ of functions rapidly decreasing at infinity, then there exists a constant $C$ such that $|\phi(x)| \leqslant C /\left(1+x^{2}\right)$ for all $x$. Then

$$
\begin{aligned}
\sum_{n=-\infty}^{\infty}\left|\phi\left(x-n h^{-1}\right)\right| & \leqslant C \sum_{n=-\infty}^{\infty}\left(1+\left(x-n h^{-1}\right)^{2}\right)^{-1} \\
& \leqslant 8 C \sum_{n=-\infty}^{\infty} \frac{\left(1+x^{2}\right)}{1+\left(n h^{-1}\right)^{2}}<\infty .
\end{aligned}
$$

Thus $\sum_{n=-\infty}^{\infty}\left|\phi\left(x-n h^{-1}\right)\right|$ converges absolutely to a function $\Phi(x)$ which is periodic, continuous and hence bounded. Thus

$$
\begin{aligned}
\sum_{n=-\infty}^{\infty} \int\left|\hat{g}_{n}(x) \phi(x)\right| d x & =\sum_{n=-\infty}^{\infty} \int\left|\hat{g}(x) \phi\left(x-n h^{-1}\right)\right| d x \\
& =\int \sum_{n=-\infty}^{\infty}\left|\hat{g}(x) \phi\left(x-n h^{-1}\right)\right| d x=\int|\hat{g}(x)||\Phi(x)| d x .
\end{aligned}
$$

Now $\Phi$ is bounded and a support of $\hat{g}$ is $\Lambda_{0}$ which has finite measure (Lloyd [5]) so $\hat{g}$ is actually an $L_{1}(\mathbf{R})$ function and the above integral is finite. Thus $\sum_{n=-\infty}^{\infty} \hat{g}_{n}(\phi)$ is finite and

$$
\begin{aligned}
\sum_{n=-\infty}^{\infty} \hat{g}_{n}(\phi) & =\sum_{n=-\infty}^{\infty} \int \hat{g}(x) \phi\left(x-n h^{-1}\right) d x \\
& =\int \hat{g}(x) \sum_{n=-\infty}^{\infty} \Phi\left(x-n h^{-1}\right) d x
\end{aligned}
$$

and so $\sum_{n=-\infty}^{\infty} \hat{g}_{n}$ converges to a temperate distribution $G$ say, which is periodic, with period $h^{-1}$. Expanding $G$ in its Fourier series, $G=\Sigma c_{n} e^{-2 \pi i n h x}$, where the Fourier coefficient $c_{n}$ is given by $c_{n}=h G\left(\xi(x) e^{-2 \pi i n h x}\right)$ where $\xi$ is a unitary function, i.e. a test function satisfying $\sum_{n=-\infty}^{\infty} \xi(t-n h)=1$ for all $t \in \mathbf{R}$ (see e.g. Zemanian $[9, \$ 11.6]$ ). Now, 


$$
\begin{aligned}
G\left(\xi(x) e^{2 \pi i n h x}\right) & =\sum_{m=-\infty}^{\infty} \hat{g}_{m}\left(\xi(x) e^{2 \pi i n h x}\right) \\
& =\sum_{m=-\infty}^{\infty} \int \hat{g}\left(x-m h^{-1}\right) \xi(x) e^{2 \pi i n h x} d x \\
& =\sum_{m=-\infty}^{\infty} \int \hat{g}(x) \xi\left(x+m h^{-1}\right) e^{2 \pi i n h\left(x+m h^{-1}\right)} d x \\
& =\int \hat{g}(x) \sum_{m=-\infty}^{\infty} \xi\left(x+m h^{-1}\right) e^{2 \pi i n h x} d x \\
& =\int \hat{g}(x) e^{2 \pi i n h x} d x=g(n h)=0
\end{aligned}
$$

so $G=0$. Suppose that $\hat{g} \neq 0$. Then there is an open set $P \subseteq \Lambda$ with $\int_{P}|g(x)|^{2} d x>0$. Let $\phi$ be a testfunction supported by $P$, then $\hat{g}(\phi(x-$ $\left.n h^{-1}\right)$ ) $=0$ for $x \in P$ and $n \neq 0$, since the translates of $P$ are disjoint. Thus $\hat{g}(\phi)=G(\phi)=0$. But $\mathscr{Q}(P)$ is dense in $L_{2}(P)$ (Trèves [10, p. 159]), contradicting the hypothesis that $\hat{g} \neq 0$. Thus $\hat{g}$ and hence $g$ is zero, and so $H(R)=\mathfrak{N}_{R}$. Because of the isomorphism between $H(X)$ and $H(R)$, it also follows that $H(X)=\Re$ and so $X(t)$ is determined by its samples.

We now turn to the development of interpolation formulae that will exactly reconstruct the process from its samples, when $H(X)=\mathfrak{N}$. We first consider a lemma which will prove useful.

LEMMA 1. Let $f \in L_{2}(\mathbf{R})$ and suppose that there exists an open set $Q_{0}$ such that the translates of $Q_{0}$ by $n h^{-1}$ are all disjoint and $Q_{0} \supseteq \operatorname{supp} \hat{f}$. Let $W$ be an open set such that supp $\hat{f} \subseteq W \subseteq \bar{W} \subseteq Q_{0}$ and let $\psi$ be $a C^{\infty}$ function that is 1 on $\bar{W}$ and 0 on $\mathrm{C} Q_{0}$. Let $K(t)$ be the function $K(t)=h \int e^{2 \pi i t x} \psi(x) d x$. Then, the sequence $\sum_{n=-N}^{N} K(t-n h) f(n h)$ converges uniformly to $f(t)$.

Proof. Since the translates of $Q_{0}$ are disjoint, the Lebesgue measure of $Q_{0}$ is finite (Lloyd [5, Corollary to Theorem 1]). Thus $\hat{f} \in L_{1} \cap L_{2}$ by the Hölder inequality. Thus $f$ is continuous and is given by $f(t)=\int_{W} e^{2 \pi i t x} \hat{f}(x) d x$. Define the function $F_{t}(x)=\sum_{n=-\infty}^{\infty} \psi\left(x+n h^{-1}\right) e^{2 \pi i\left(x+n h^{-1}\right) t} . F_{t}(x)$ is $C^{\infty}$ and periodic, so it is the limit of its Fourier series $\Sigma_{n} K(t-n h) e^{2 \pi i n h x}$ which converges uniformly.

Thus if we set $\varepsilon_{N}(t)=\sup _{x}\left|F_{t}(x)-\sum_{n=-N}^{N} K(t-n h) e^{2 \pi i n h x}\right|$ then $\lim _{N} \varepsilon_{N}(t)=0$ for each $t$ and

$$
\begin{aligned}
\left|\int_{\bar{W}} F_{t}(x) \hat{f}(x) d x-\sum_{n=-N}^{N} \int_{\bar{W}} K(t-n h) e^{2 \pi i n h x} \hat{f}(x) d x\right| \\
\quad<\int_{\bar{W}} \varepsilon_{N}(t)|\hat{f}(x)| d x \leqslant \varepsilon_{N}(t)\|f\|_{L_{2}} m(\bar{W})^{1 / 2}
\end{aligned}
$$


by the Plancherel Theorem and the Cauchy-Schwartz inequality and where $m$ denotes Lebesgue measure.

But $F_{t}(x)=e^{2 \pi i t x}$ on $\bar{W}$, so (4) implies $f(t)=\lim _{N \rightarrow \infty} \Sigma_{n=-N}^{N} K(t-$ $n h) f(n h)$, which proves the lemma. We can now generalise Theorem 3 of Lloyd [5]:

THEOREM 2. Suppose $\{X(t), t \in \mathbf{R}\}$ is a random process of the type considered at the beginning of $\$ 2$. Suppose that $Q$ is an open support of $\hat{R}$ whose translates by $\left(n h^{-1}, n h^{-1}\right)$ are all disjoint. Then

$$
X(t)=\lim _{N \rightarrow \infty} \sum_{n=-N}^{N} X(n h) K(t-n h), \quad-\infty<t<\infty,
$$

where $K$ is the function defined in Lemma 1.

Proof. In view of the isomorphism between $H(X)$ and $H(R)$, it is enough to prove that the function $R(\cdot, t)$ in $H(R)$ is the limit in $H(R)$ of the sequence $\sum_{n=-N}^{N} R(\cdot, n h) K(t-n h)$. Now

$$
\begin{aligned}
\| R(\cdot, t)- & \sum_{n=-N}^{N} R(\cdot, n h) K(t-n h) \|_{H(R)}^{2} \\
& =\sum_{j} \lambda_{j}\left|f_{j}(t)-\sum_{n=-N}^{N} f_{j}(n h) K(t-n h)\right|^{2} .
\end{aligned}
$$

Now let $W$ be an open set such that for all $j$, supp $f_{j} \subseteq W \subseteq \bar{W} \subseteq Q_{0}=\{x$ : $(x, x) \subseteq Q\}$. Such a set exists because for each $j$, supp $\hat{\hat{f}}_{j}=\{x:(x, x) \in$ Supp $\hat{R}\} \subseteq Q_{0}$ and $Q_{0}$ is open. $Q_{0}$ has disjoint translates by $h^{-1}$, so by using the notation and method of Lemma $1,(6)$ is less than $\Sigma_{j} \lambda_{j}\left\|f_{j}\right\|_{L_{2}} \varepsilon_{N}(t) m(\bar{W})^{1 / 2}$. The $f_{j}$ are orthonormal in $L_{2}, \Sigma \lambda_{j}<\infty$ since $R$ is trace class and $m(\bar{W})<\infty$. Thus (6) converges to zero for every $t \in \mathbf{R}$, proving the theorem.

The final theorem in this section shows that the sampling series (5) converges almost surely as well as in mean square.

THEOREM 3. Under the hypotheses of Theorem 2, the sampling series (5) converges almost surely.

Proof. By Theorem 7 of Cambanis and Masry [7] the representation (2) of the process $x(t)$ converges in $L_{2}$ almost surely, so if $x_{N}(t, \omega)$ is defined by $x_{N}(t, \omega)=\sum_{j=1}^{N} f_{j}(t) e_{j}(\omega)$ then $x_{N}(t, \omega)$ converges in $L_{2}$ to $x(t, \omega)$ for almost all $\omega . \hat{x}_{N}(\lambda, \omega)$ has support in $Q_{0}=\{\lambda:(\lambda, \lambda) \in Q\}$ for each $N$ since each of the functions $\hat{f}_{j}(\lambda)$ is supported by $Q_{0}$, so $\hat{x}(\lambda, \omega)$ is supported by $Q_{0}$. Thus for almost all $\omega, x(t, \omega)$ satisfies the hypotheses of Lemma 1 and so (5) converges almost surely. 
3. Extension of the sampling theorem. In this section we extend the results of $\S 2$ to include processes $\{X(t), t \in \mathbf{R}\}$ which satisfy

$$
\int R(t, t) \mu_{k}(d t)<\infty
$$

for some positive integer $k$, where $\mu_{k}(d t)=\left(1+t^{2}\right)^{-k} d t$. If (7) is satisfied, then $R(t, s)=E(X(t) \overline{X(s)})$ satisfies

$$
\iint \frac{|R(t, s)|^{2}}{\left(1+t^{2}+s^{2}\right)^{2 k}} d t d s<\infty
$$

since $\left(1+t^{2}+s^{2}\right)^{2} \geqslant\left(1+t^{2}\right)\left(1+s^{2}\right)$. It follows that the Fourier transform of $R(t, s)$ exists as a distribution in the Sobolev space $H^{2,-2 k}\left(\mathbf{R}_{2}\right)$ (see, e.g., Trèves [10, Chapter 31]). The following theorem is an extension of Theorem 1.

THEOREM 4. Let $\{X(t), t \in \mathbf{R}\}$ be a second order measurable mean square continuous random process whose covariance $R$ satisfies (7). Let $Q$ be an open set such that supp $\hat{R} \subseteq Q$ and suppose that the sets $\left(n h^{-1}, n h^{-1}\right)+Q$ are disjoint for all integers $n$. Then the process is determined by its samples $\{X(n h), n \in \mathbf{Z}\}$.

Proof. Consider the operator $R: L_{2}\left(\mu_{k}\right) \rightarrow L_{2}\left(\mu_{k}\right)$ with kernel $R$. Letting $\lambda_{j}$ be the nonzero eigenvalues of $R$ with corresponding eigenvectors $e_{j}$, we see that the RKHS corresponding to $X(t)$ is exactly the same as in $\$ 2$, except that the $\lambda_{j}$ now are eigenvalues of an operator on $L_{2}\left(\mu_{k}\right)$. instead of $L_{2}(\mathbf{R})$ $\left(=L_{2}\left(\mu_{0}\right)\right)$. To prove the theorem it is enough to show that if $\left\{g_{j}\right\}$ is a sequence with $\Sigma_{j} \lambda_{j} f_{j}(n h) \bar{g}_{j}=0$ and $\Sigma_{j} \lambda_{j}\left|g_{j}\right|^{2}<\infty$ then $g_{j}=0$ for all $j$. Here the functions $f_{j}$ are the "time functions" for $X(t)$ and are functions in $L_{2}\left(\mu_{k}\right)$. The process $X(t)$ still has the representation (2), with the $f_{j}$ and $\lambda_{j}$ as above. Let $g(t)=\Sigma_{j} \lambda_{j} f_{j}(t) \bar{g}_{j}$. Then $g \in L_{2}\left(\mu_{k}\right)$, and $\hat{g}$ is a distribution in $H^{2,-k}(\mathbf{R})$ given by $\hat{g}(\phi)=\Sigma_{j} \lambda_{j} \hat{f}_{j}(\phi) \bar{g}_{j}$. Now

$$
|\hat{g}(\phi)|^{2} \leqslant \sum_{j} \lambda_{j}\left|\hat{f}_{j}(\phi)\right|^{2} \sum_{j} \lambda_{j}\left|g_{j}\right|^{2} \leqslant \sum_{j} \lambda_{j}\left|g_{j}\right|^{2} \hat{R}(\phi \otimes \bar{\phi})
$$

and an argument similar to that employed in Theorem 1 shows that supp $\hat{g} \subseteq$ $Q_{0}$ and the sets $n h+Q_{0}$ are disjoint for each $n$, where $Q_{0}=(x:(x, x) \in Q)$ as before. Now since $\hat{g} \in H^{2,-k}(\mathbf{R})$, we may write $\hat{g}=\sum_{m=0}^{k}(-1)^{m} D^{2 m} \hat{u}$ where $D$ is a differentiation operator $d^{m} / d x^{m}$ and $\hat{u} \in H^{2, k}(\mathbf{R})$. Moreover, we can suppose that supp $\hat{u} \subseteq Q_{0}$ (Trèves [10, Chapters 24, 31]). Now consider the sum $\Sigma_{n=-N}^{N} \mathcal{T}_{n} \hat{g}$, where $\mathscr{T}_{n}$ is the translation operator on $\mathscr{D}^{\prime}$ defined by $\left(\mathscr{T}_{n} \hat{g}\right)(\phi)=g\left(\mathscr{T}_{n} \phi\right)$, and also the translation operator on $\mathscr{D}$ defined by $\mathscr{T}_{n} \phi(t)=\phi(t-n h)$. Now $\mathcal{T}_{n} D^{m} u=D^{m} \mathcal{T}_{n} u$ so 


$$
\sum_{n=-N}^{N} \mathcal{T}_{n} \hat{g}=\sum_{n=-N}^{N} \mathcal{T}_{n} \sum_{m=0}^{k}(-1)^{m} D^{2 m} \hat{u}=\sum_{m=0}^{k}(-1)^{m} D^{2 m} \sum_{n=-N}^{N} \mathcal{T}_{n} \hat{u} .
$$

Since $\hat{u} \in H^{2, k}(\mathbf{R})$, by the proof of Theorem $1 \sum_{n=-N}^{N} \mathcal{T}_{n} \hat{u}$ converges to a periodic distribution. Thus $\Sigma \mathcal{T}_{n} \hat{g}$ converges to a periodic distribution $G$, whose Fourier expansion is $G=\sum_{n=-\infty}^{\infty} g(n h) e^{-2 \pi i n h x}$. But $g(n h)=0$ so $G=0$. Now supp $\hat{g}$ is a closed set contained in $Q_{0}$, so supp $\hat{g}$ and $\mathcal{C} Q_{0}$ are disjoint closed sets, and there exists an open set $W$ with supp $\hat{g} \subseteq W \subseteq \bar{W} \subseteq$ $Q_{0}$. Let $\psi$ be a $C^{\infty}$ function that is 1 on $\bar{W}$ and 0 on $C Q_{0}$. Then for any $\xi \in \mathscr{D}, \xi=(1-\psi) \xi+\psi \xi . \psi \xi$ is supported by $Q_{0}$ so $G(\psi \xi)=\hat{g}(\psi \xi)$, and $\hat{g}((1-\psi) \xi)=0$ since the support of $(1-\psi) \xi$ is disjoint from supp $\hat{g}$. Thus $g(\xi)=G(\psi \xi)+\hat{g}((1-\psi) \xi)=0$ so $\hat{g}=0$ and thus $g=0$. It follows that $H(R)=\mathfrak{N}_{R}$ and so $H(X)=\mathfrak{R}$.

Next we present a sampling theorem for processes whose covariances satisfy the hypotheses of the last theorem.

THEOREM 5. Let $\{X(t), t \in \mathbf{R}\}$ be a second order random process satisfying the hypotheses of Theorem 4 . Then the sequence

$$
\sum_{n=-N}^{N} X(n h) K(t-n h)
$$

converges to $X(t)$ in mean square for every $t \in \mathbf{R}$, where $K$ is the function defined in Lemma 1.

Proof. As in the proof of Theorem 2, it is enough to show that

$$
\lim _{N} \sum_{n=-N}^{N} f_{j}(n h) K(t-n h)=f_{j}(t), \quad j=1,2, \ldots,
$$

uniformly in $j$ for each $t$. The Fourier transform of each $f_{j}$ is a distribution in $H^{2,-k}(\mathbf{R})$, so for each $j, \hat{f}_{j}=\sum_{m=0}^{k}(-1)^{m} D^{2 m} \hat{u}_{j}$ and $f_{j}(t)=$ $\sum_{m=0}^{k}(-1)^{m}(2 \pi i t)^{2 m} u_{j}(t)$ for some function $u_{j}$ in $L_{2}$ whose Fourier transform $\hat{u}_{j}$ is in $H^{2, k}(\mathbf{R})$ supported by $Q_{0}$. Thus it is enough to prove that for $0 \leqslant m \leqslant k$,

$$
\lim _{N} \sum_{n=-N}^{N} K(t-n h)(2 \pi i t)^{2 m} u_{j}(n h)=(2 \pi i t)^{2 m} u_{j}(t)
$$

uniformly in $j$. Each $\hat{u}_{j}$ is in $L_{1} \cap L_{2}$ since $Q_{0}$ has finite Lebesgue measure, so $u_{j}(t)=\int_{\Gamma_{j}} e^{2 \pi i t x} \hat{u}_{j}(x) d x$ where $\Gamma_{j}=\operatorname{supp} u_{j} \subseteq Q_{0}$. Let $\psi$ be the $C^{\infty}$ function that is 1 on $\bar{W}$ and 0 on $e Q_{0}$ where now $\Gamma_{j} \subseteq W \subseteq \bar{W} \subseteq Q_{0}$ for all $j$, and define $F_{t}(x)$ as in the proof of Lemma 1. $F_{t}$ is $C^{\infty}$ with period $h^{-1}$, its Fourier series $\sum_{n=-\infty}^{\infty} K(t-n h) e^{2 \pi i n h x}$ converges uniformly on $\mathbf{R}$ to $F_{t}(x)$, and the differentiated series $\sum_{n=-\infty}^{\infty} K(t-n h)(2 \pi i n h)^{2 m} e^{2 \pi i n h x}$ converge uniformly to $d^{2 m} F_{t}(x) / d x^{2 m}$. On $W, d^{2 m} F_{t}(x) / d x^{2 m}=(2 \pi i t)^{2 m} e^{2 \pi i t x}$ so, on $W$, 
$\lim _{N} \sum_{n=-N}^{N} K(t-n h)(2 \pi i n h)^{2 m} e^{2 \pi i n h x}$ converges uniformly to $(2 \pi i t)^{2 m} e^{2 \pi i t}$. Moreover

$$
\begin{aligned}
& \left|\sum_{n=-N}^{N} K(t-n h)(2 \pi i n h)^{2 m} u_{j}(n h)-(2 \pi i t)^{2 m} u_{j}(t)\right| \\
& \quad<\int_{\Gamma_{j}}\left|\sum_{n=-N}^{N} K(t-n h)(2 \pi i n h)^{2 m} e^{2 \pi i n h x}-(2 \pi i t)^{2 m} e^{2 \pi i t x}\right|\left|\hat{u}_{j}(x)\right| d x \\
& \leqslant \sup _{x \in W}\left|\sum_{n=-N}^{N} K(t-n h)(2 \pi i n h)^{2 m} e^{2 \pi i n h x}-(2 \pi i t)^{2 m} e^{2 \pi i t x}\right| m\left(Q_{0}\right)^{1 / 2}\left\|u_{j}\right\|_{L_{2}(\mathrm{R})} \\
& <K_{N, m}(t) m\left(Q_{0}\right)^{1 / 2}\left\|u_{j}\right\|_{L_{2}(\mathrm{R})} \text { say, }
\end{aligned}
$$

where $\lim _{N} K_{N, m}(t)=0$ for $m=0,1, \ldots, k$, and $m$ denotes Lebesgue measure. Now let $\varepsilon>0$, then for $N$ sufficiently large

$$
\begin{aligned}
\left|f_{j}(t)-\sum_{n=-N}^{N} K(t-n h) f_{j}(n h)\right| & \leqslant \sum_{m=0}^{k} K_{N, m}(t) m\left(Q_{0}\right)^{1 / 2}\left\|u_{j}\right\|_{L_{2}(\mathbf{R})} \\
& <\varepsilon m\left(Q_{0}\right)^{1 / 2}\left\|u_{j}\right\|_{L_{2}(\mathbf{R})} .
\end{aligned}
$$

Also $f_{j}(t)=\left(\sum_{m=0}^{k}(2 \pi t)^{2 m}\right) u_{j}(t)$ so

$$
\begin{aligned}
\int\left|f_{j}(t)\right|^{2} & \left(1+t^{2}\right)^{-k} d t \\
= & \int\left(\sum_{m=0}^{k}(2 \pi t)^{2 m}\right)^{2}\left(1+t^{2}\right)^{-k}\left|u_{j}(t)\right|^{2} d t \geqslant C^{2}\left\|u_{j}\right\|_{L_{2}(K)}^{2}
\end{aligned}
$$

for some constant $C>0$. The time functions $f_{j}$ are orthonormal in $L_{2}\left(\mu_{k}\right)$ so finally we obtain $\left\|u_{j}\right\|_{L_{2}(k)} \leqslant C^{-1}$ for all $j$ and $\mid f_{j}(t)-\Sigma_{n=-N}^{N} K(t-$ $n h) f_{j}(n h) \mid \leqslant \varepsilon m\left(Q_{0}\right)^{1 / 2} C^{-1}$ for all $j$. Thus (9) is verified.

The next theorem shows that the sequence (8) converges almost surely:

THEOREM 6. Let $\{X(t), t \in \mathbf{R}\}$ be a random process satisfying the hypotheses of Theorem 5 . Then for almost every $\omega$,

$$
\lim _{N} \sum_{n=-\infty}^{\infty} X(n h, \omega) K(t-n h)=X(t, \omega)
$$

for each $t \in \mathbf{R}$.

Proof. By Cambanis and Masry [7, Theorem 2], $X(t, \omega)$ is a function in $L_{2}\left(\mu_{k}\right)$ for almost all $\omega$, so its Fourier transform is in $H^{2,-k}(\mathbf{R})$. It is enough to prove that the support of $\hat{X}(x, \omega)$ is in the set $Q_{0}$, then the argument of the last theorem will prove the theorem. By Theorem 7, Cambanis and Masry, the r.v.'s $X_{N}(t, \omega)=\sum_{j=1}^{N} f_{j}(t) e_{j}(\omega)$ converge almost surely in $L_{2}\left(\mu_{k}\right)$ to $X(t, \omega)$, 
so for any $\phi \in S, \hat{X}_{N}(\phi, \omega)$ converges to $\hat{X}(\phi, \omega)$ for almost all $\omega$ since

$$
\begin{aligned}
\left|\hat{X}_{N}(\phi, \omega)-\hat{X}(\phi, \omega)\right|^{2} & \leqslant\left|\int\left(X_{N}(t, \omega)-X(t, \omega)\right) \hat{\phi}(t) d t\right|^{2} \\
& <\int\left|X_{N}(t, \omega)-X(t, \omega)\right|^{2} \mu_{k}(d t) \int\left(1+t^{2}\right)^{k}|\hat{\phi}(t)|^{2} d t
\end{aligned}
$$

by the Cauchy-Schwartz inequality.

Now suppose that $x \notin Q_{0}$, so that $(x, x) \notin Q$, the open support of $\hat{R}$ whose translates are disjoint. It follows that there is an open neighbourhood $U$ of $(x, x)$ such that all testfunctions in $\mathscr{D}\left(\mathbf{R}_{2}\right)$ supported by $U$ are mapped by $\hat{R}$ onto 0 . Let $V$ be an open neighbourhood of $x$ with $V \times V \subseteq U$, then for any $\phi$ in $\mathscr{D}\left(\mathbf{R}_{1}\right)$ supported by $V, \sum_{j=1}^{\infty} \lambda_{j}\left|\hat{f}_{j}(\phi)\right|^{2}=\hat{R}(\phi \otimes \phi)=0$. Thus $\hat{f}_{j}(\phi)=0$ for all $j$ and so $\hat{X}_{N}(\phi, \omega)=0$ for all $N$, and hence $\hat{X}(\phi, \omega)=0$. Consequently for any $\phi \in \mathscr{D}\left(\mathbf{R}_{1}\right)$ supported by $V, \hat{X}(\phi, \omega)=0$; this implies that supp $\hat{X}(\cdot, \omega) \subseteq Q_{0}$, proving the theorem.

4. Harmonizable processes. First, we present a counterexample to show that a process need not have a covariance whose Fourier transform has disjoint translates in order to be determined by its samples. Consider the very simple example of a process of the form $x(t)=f(t) e$ where $f(t)$ is a real valued function and $e$ a random variable with $E(e)=0, E|e|^{2}=1$. Then the covariance function $R$ of $x(t)$ is $R(t, s)=f(t) \overline{f(s)}$. Suppose, in addition, that the function $f$ is given by

$$
f(t)=\int_{-\infty}^{\infty} e^{2 \pi i t x} \xi(x) d x
$$

for some $L_{1}$ function $\xi \neq 0$. Then $R$ has the representation

$$
R(t, s)=\int_{-\infty}^{\infty} \int_{-\infty}^{\infty} e^{2 \pi i(t x-s y)} \xi(x) \overline{\xi(y)} d x d y
$$

so $R$ (and hence $X(t)$ ) is harmonizable.

Now the samples $\{X(n h), n \in \mathbf{Z}\}$ generate $H(X)$ if and only if $f(n h) \neq 0$ for some $n$, since $H(X)=\{\lambda e: \lambda \in \mathbf{C}\}$. Choose $\xi(x)=e^{-|x|}$ then supp $\hat{R}=$ $\operatorname{supp} e^{-(|x|+|y|)}=\mathbf{R}_{2}$ and $f(t)=\left(1+t^{2}\right)^{-1}$. Thus $f(n h) \neq 0$ for all $n$ and so $X(t)$ is determined by its samples. But the translates of $\mathbf{R}_{2}$ are certainly not disjoint, so no condition on the translates of $\hat{R}$ can be necessary.

Similar processes having corresponding spaces $H(X)$ of any finite dimension can be constructed to provide similar counterexamples. This example shows that the necessary half of Proposition 2 of Rao [6] is incorrect. It should be noted that these counterexamples are all finite dimensional. It would be interesting to see if a counterexample exists in the infinite dimensional case. However, any harmonizable process satisfies the conditions of Theorem 4 with $k=1$, so Theorem 4 is a generalisation of Rao's theorem. In 
fact, Theorem 4 holds for any harmonizable process indexed by more general types of topological groups. Suppose that $G$ is a locally compact abelian group, with character group $\hat{G}$. A random process $\{X(g), g \in G\}$ is harmonizable if it has a covariance of the form

$$
R(g, h)=E(X(g) \overline{X(h)})=\int_{\hat{G}} \int_{\hat{G}}\langle\alpha, g\rangle \overline{\langle\beta, h\rangle} \mu(d \alpha, d \beta)
$$

where $\langle\alpha, g\rangle$ denotes the character $\alpha$ evaluated at $g$ and $\mu$ is a measure in $M(\hat{G} \times \hat{G})$ satisfying the condition $\sum_{i=1}^{n} \sum_{j=1}^{n} c_{i} \bar{c}_{j} \mu\left(\Delta_{i} \times \Delta_{j}\right) \geqslant 0$ for all complex numbers $c_{1}, \ldots, c_{n}$ and Borel subsets $\Delta_{1}, \ldots, \Delta_{n}$ of $\hat{G}$. Assume also that $G$ contains a closed subgroup $H$, which plays the role of the subgroup $\{n h$ : $n \in \mathbf{Z}\}$ in $\mathbf{R}$ in the sampling theorems of previous sections. Let $A$ be the annihilator of $H$ in $\hat{G}, A=\{\alpha \in \hat{G}:\langle\alpha, h\rangle=1 \forall h \in H\}$. We seek a condition sufficient for the process $X(g)$ to be determined by its samples $\{X(g), g$ $\in H\}$ as in Theorems 1 and 4 . We must first prove a lemma.

LEMMA 2. Let $\nu$ be a regular Borel measure of finite variation on $\hat{G}$, and $\hat{\nu}$ its Fourier transform, $\hat{\nu}(g)=\int_{\hat{G}}\langle\alpha, g\rangle \nu(d \alpha)$. Then if the support $\Lambda$ of $\nu$ is disjoint from all translates of $\Lambda$ by members of $A$ not equal to the identity of $A$, and $\hat{\nu}(h)=0$ for all $h \in H$, then $\nu=0$.

Proof. Let $T$ denote the natural homomorphism $\hat{G} \rightarrow \hat{G} / A, T(\alpha)=\alpha+$ $A$. By Rudin [11, p. 53], the restriction of $\hat{v}$ to $H$ is the Fourier transform of a measure $\sigma$ on $\hat{G} / A$ such that for all bounded Borel functions $\phi$ on $\hat{G} / A$

$$
\int_{\hat{G}} \phi(T(\alpha)) \nu(d \alpha)=\int_{\hat{G} / A} \phi d \sigma .
$$

Since the restriction of $\hat{\nu}$ to $H$ is the zero function, it follows that $\sigma=0$ and

$$
\int_{\hat{G}} \phi(T(\alpha)) \nu(d \alpha)=0
$$

for all bounded Borel $\phi$ on $\hat{G} / A$. Let $\Phi(\alpha)=\Sigma_{\gamma \in A} \chi_{\Lambda}(\alpha+\gamma)$ where $\chi_{\Lambda}$ is the indicator of $\Lambda$. Since for $\alpha_{1} \in \alpha+A, \Phi\left(\alpha_{1}\right)=\Phi(\alpha)$, we can regard $\Phi$ as a Borel function on $\hat{G} / A$ which is bounded since the translates of $\Lambda$ are disjoint. Thus $\Phi$ satisfies (10). Moreover $\Phi \circ T=1$ on $\Lambda$, so that

$$
\nu(\Lambda)=\int_{\Lambda} 1 \nu(d \alpha)=\int_{\Lambda} \Phi(T(\alpha)) \nu(d \alpha)=\int_{\hat{G}} \Phi(T(\alpha)) \nu(d \alpha)=0 .
$$

It follows that $\nu=0$.

Now consider the linear space $\mathcal{L}$ consisting of all functions $f$ on $\hat{G}$ such that the integral $\int_{\hat{G}} \int_{\hat{G}} f(\alpha) \overline{f(\beta)} \mu(d \alpha, d \beta)$ exists, where $\mu$ is the measure in the representation of $R(g, h)$. If we identify functions $f_{1}$ and $f_{2}$ for which

$$
\int_{\hat{G}} \int_{\hat{G}}\left(f_{1}(\alpha)-f_{2}(\alpha)\right) \overline{\left(f_{1}(\beta)-f_{2}(\beta)\right)} \mu(d \alpha, d \beta)=0,
$$


we may define an inner product $\left(f_{1}, f_{2}\right)_{\mathfrak{L}}$ on $\mathcal{L}$ by

$$
\left(f_{1}, f_{2}\right)_{\mathfrak{L}}=\int_{\hat{G}} \int_{\hat{G}} f_{1}(\alpha) \overline{f_{2}(\beta)} \mu(d \alpha, d \beta) ;
$$

the resulting inner product space can be completed to give a Hilbert space $\Lambda_{2}(\mu)$ with inner product $\left(f_{1}, f_{2}\right)_{\Lambda}$ and norm $\|f\|_{\Lambda}$. For functions $f_{1}, f_{2}$ in $\mathcal{E}$, of course $\left(f_{1}, f_{2}\right)_{\mathcal{L}}=\left(f_{1}, f_{2}\right)_{\Lambda}$. The Hilbert spaces $\Lambda_{2}(\mu)$ and $H(X)$ are isomorphic under the correspondence $X(g) \leftrightarrow g$, where $g$ is the function in $\mathcal{L}$ given by $g(\alpha)=\langle\alpha, g\rangle, g \in \mathcal{G}$. (See e.g. Cambanis and Liu [12]). The samples $\{X(h), h \in H\}$ generate $H(X)$ if and only if the space $\Lambda_{2}(\mu)$ is generated by the functions $h, h \in H$. The analog of Theorems 1 and 4 is

THEOREM 7. If the support $S$ of $\mu$ has its translates by $(\gamma, \gamma)$ disjoint for all $\gamma \in A$, then the samples $\{X(h), h \in H\}$ generate $H(X)$.

Proof. It is enough to prove that the functions $h, h \in H$, generate $\Lambda_{2}(\mu)$. Let $f \in \Lambda_{2}(\mu)$ and suppose that $(f, h)_{\Lambda}=0$ for all $h \in H$. We will show that $f=0$. Define a measure $\nu$ on the Borel sets of $\hat{G}$ by $\nu(\Delta)=\left(\chi_{\Delta}, f\right)_{\Lambda}$ where $\chi_{\Delta}$ is the indicator function of the Borel set $\Delta$. Then

$$
\begin{aligned}
|\nu(\Delta)| & =\left|\left(\chi_{\Delta}, f\right)\right|_{\Lambda} \leqslant\left\|\chi_{\Delta}\right\|_{\Lambda}\|f\|_{\Lambda} \\
& =\left(\chi_{\Delta}, \chi_{\Delta}\right)_{\mathcal{L}}^{1 / 2}\|f\|_{\Lambda}=\mu(\Delta \times \Delta)^{1 / 2}\|f\|_{\Lambda}
\end{aligned}
$$

so $\nu$ is a finite measure. Now $S$, the support of $\mu$, is given by $S=\mathcal{U} \cup\{: U$ open, $\mu(U)=0\}$ so if $(\alpha, \alpha) \notin S$ then there is an open set $U$ containing $(\alpha, \alpha)$ with $\mu(U)=0$. Since $U$ is open, there is an open subset $V$ of $\hat{G}$ with $\alpha \in V, V \times V \subseteq U$, and $\mu(V \times V)=0$, thus $\nu(E)=\left(\chi_{E}, f\right)_{\Lambda} \leqslant \mu(V \times$ $V)^{1 / 2}\|f\|_{\Lambda}=0$ and $\alpha \notin \operatorname{supp} \nu$. Thus $\{\beta:(\beta, \beta) \in S\} \supseteq \operatorname{supp} \nu$, and the translates of supp $\nu$ by elements $\gamma$ in $A$ are disjoint. Moreover $\hat{\nu}(h)=$ $\int\langle\alpha, h\rangle \nu(d \alpha)=(h, f)_{\Lambda}=0$ for all $h \in H$, so by Lemma $2 \hat{\nu}=0$, and hence $\left(\chi_{\Delta}, f\right)_{\Lambda}=0$ for all Borel sets $\Delta$. But the functions $\chi_{\Delta}$ are dense in $\Lambda_{2}(\mu)$ so $f=0$, and thus the functions $h, h \in H$, generate $\Lambda_{2}(\mu)$.

If we assume a little more about the subgroup $H$, we can develop a sampling theorem for $X(g)$. Suppose that $H$ is now an infinite closed discrete finitely generated subgroup with generator $h_{0}$; such will exist in $G$, for example, if $G$ contains an element $h_{0}$ such that the smallest closed subgroup containing $h_{0}$ is not compact (Hewitt and Ross [13, p. 84]). Since $H$ is discrete, its character group $\hat{H}$ will be compact and hence $\hat{G} / A$ which is isomorphic to $\hat{H}$ will also be compact. Under these conditions the following theorem is true:

THEOREM 8. There exists a sequence $S_{n}$ of functions on $H$ such that

(i) $S_{n}$ has finite support for each $n$,

(ii) $\sup _{h \in H}\left|S_{n}(h)\right| \leqslant 1$ for each $n$, 
(iii) $\lim _{n \rightarrow \infty} S_{n}(h)=1$ if $h=e$ and zero otherwise. If $\in L_{1}(\hat{G} / A)$ then

$$
\lim _{n \rightarrow \infty} \sum_{h \in H} S_{n}(h) \hat{f}(h)\langle\alpha, h\rangle=f(T(\alpha))
$$

for every $\alpha \in \hat{G}$ such that $f$ is continuous at $T(\alpha)$. The series (11) converges uniformly on $\hat{G} / A$ if $f$ is continuous.

Proof. The proof is a specialization to the present context of Theorem 7.1 of Mayer [14], and is omitted. Note that the Fourier transform of $f$ is a function on $H$ since $(\hat{G} / A)^{\wedge}$ is isomorphic to $H$.

Note. If $G=\mathbf{R}$ and $H=\mathbf{Z}$ the above theorem is just the de la Vallee-Poisson method of summation of a Fourier series.

We can now give the sampling expansion of harmonizable process:

THEOREM 9. Suppose $H$ is an infinite closed discrete cyclic subgroup of an LCA group G. Suppose $\{X(g), g \in G\}$ is a harmonizable process with spectral measure $\mu$ with an open support $Q$ whose translates by members of $A$ (the annihilator of $H$ in $\hat{G})$ are all disjoint. Let $W$ be an open set such that $\{\alpha$ : $(\alpha, \alpha) \in \operatorname{Supp} \mu\} \subseteq W \subseteq \bar{W} \subseteq\{\alpha:(\alpha, \alpha) \in Q\}$, and let $\psi$ be a continuous bounded function on $\hat{G}$ that is equal to unity on $\bar{W}$ and zero on $\mathcal{C}\{\alpha$ : $(\alpha, \alpha) \in Q\}$. Then there exist coefficients $a_{g}(h)$ such that

$$
X(g)=\lim _{n \rightarrow \infty} \sum_{h \in H} S_{n}(h) a_{g}(h) X(h) \text { for all } g \in G,
$$

with (12) converging in mean square.

If $A$ is discrete, then the coefficients $a_{g}(h)$ are given by

$$
a_{g}(h)=\int_{\hat{G}} \psi(\alpha)\langle\alpha, g-h\rangle M_{\hat{G}}(d \alpha)
$$

where $M_{\hat{G}}$ is the Haar measure on $\hat{G}$. In general, there is a finite measure $\nu$ on the Borel subsets of $W$ such that $a_{g}(h)=\int_{W} \psi(\alpha)\langle\alpha, g-h\rangle \nu(d \alpha)$.

Proof. Define $F_{g}(\alpha)=\Sigma_{\lambda \in A} \phi(\alpha+\lambda)\langle\alpha+\lambda, g\rangle$. Clearly we can regard $F_{g}$ as a function on $\hat{G} / A$; we claim it is continuous on $\hat{G} / A$. Let $T(\alpha)$ be a point in $\hat{G} / A$. Either $T(\alpha) \cap \bar{W}=\varnothing$ or $T(\alpha) \cap \bar{W}$ is a singleton. In the first case, there is a neighbourhood of $T(\alpha)$ in $\hat{G} / A$ on which $F_{g}(\alpha)=0$ so $F_{g}$ is continuous at $T(\alpha)$. In the second case, suppose $T(\alpha) \cap \bar{W}=\left\{\alpha_{0}\right\}$. Let $U$ be a neighbourhood of $\alpha_{0}$ in $\hat{G}$ with $U \subseteq\{\alpha:(\alpha, \alpha) \in Q\}$ such that $\mid \phi(\beta)\langle\beta, g\rangle$ $-\phi\left(\alpha_{0}\right)\left\langle\alpha_{0}, g\right\rangle \mid<\varepsilon$ for all $\beta \in U . T(U)$ is a neighbourhood of $T(\alpha)$ in $\hat{G} / A$, and for all points $T(\beta)$ in $T(U),\left|F_{g}(T(\alpha))-F_{g}(T(\beta))\right|=\mid \phi(\beta)$. $\langle\beta, g\rangle-\phi\left(\alpha_{0}\right)\left\langle\alpha_{0}, g\right\rangle \mid\langle\varepsilon$ since without loss of generality we can assume $\beta \in U$, and the translates of $\{\alpha:(\alpha, \alpha) \in Q\}$ are disjoint. Thus $F_{g}$ is continuous at $T(\alpha)$ and so continuous on $\hat{G} / A . F_{g}$ is bounded, and the Haar 
measure on $\hat{G} / A$ is finite since $\hat{G} / A$ is compact, so $F_{g} \in L_{1}(\hat{G} / A)$. Thus by Theorem $8, \Sigma_{h \in H} S_{n}(h) \hat{F}_{g}(h)\langle\alpha, h\rangle$ converges uniformly to $F_{g}(T(\alpha))$.

It follows that since $F_{g}(T(\alpha))=\langle\alpha, g\rangle$ for all $\alpha \in \bar{W}$, the series $\sum_{h \in H} S_{n}(h) \hat{F}_{g}(h)\langle\alpha, h\rangle$ converges uniformly on $\bar{W}$ to $\langle\alpha, g\rangle$. Now we claim that Supp $\mu \subseteq S_{0} \times S_{0}$, where $S_{0}=\{\alpha:(\alpha, \alpha) \in \operatorname{Supp} \mu\}$. To prove this, let $\left(\alpha_{1}, \alpha_{2}\right) \in \operatorname{Supp} \mu$. We must show that $\left(\alpha_{i}, \alpha_{i}\right) \in \operatorname{Supp} \mu$ for $i=1$, 2. Let $U_{i}$ be an arbitrary open set containing $\left(\alpha_{i}, \alpha_{i}\right)$, then there exist open neighbourhoods $V_{i}$ in $\mathrm{R}_{1}$ with $\alpha_{i} \in V_{i}$ and $V_{i} \times V_{i} \subseteq U_{i} \cdot V_{1} \times V_{2}$ is an open neighbourhood of $\left(\alpha_{1}, \alpha_{2}\right)$ so there exists a neighbourhood $V_{1}^{\prime} \times V_{2}^{\prime}$ of $\left(\alpha_{1}, \alpha_{2}\right)$ with $\mu\left(V_{1}^{\prime} \times V_{2}^{\prime}\right) \neq 0, V_{i}^{\prime} \subseteq V_{i}$. Then $V_{i}^{\prime} \times V_{i}^{\prime}, i=1,2$, are neighbourhoods of $\left(\alpha_{i}, \alpha_{i}\right)$ with $\left\{\mu\left(V_{1}^{\prime} \times V_{1}^{\prime}\right) \mu\left(V_{2}^{\prime} \times V_{2}^{\prime}\right)\right\}^{1 / 2} \geqslant\left|\mu\left(V_{1}^{\prime} \times V_{2}^{\prime}\right)\right| \neq 0$ so $\mu\left(V_{i}^{\prime} \times\right.$ $\left.V_{i}^{\prime}\right) \neq 0$, and $V_{i}^{\prime} \times V_{i}^{\prime} \subseteq U_{i}$. Thus $\left(\alpha_{i}, \alpha_{i}\right) \in \operatorname{supp} \mu$, and supp $\mu \subseteq S_{0} \times S_{0}$, proving the claim. Thus on supp $\mu$,

$$
\left(\sum_{h \in H} S_{n}(h) \hat{F}_{g}(h)\langle\alpha, h\rangle-\langle\alpha, g\rangle\right) \overline{\left(\sum_{h \in H} S_{n}(h) \hat{F}_{g}(h)\langle\beta, h\rangle-\langle\beta, g\rangle\right)}
$$

converges uniformly to 0 and hence $\left\|\Sigma S_{n}(h) \hat{F}_{g}(h)\langle\alpha, h\rangle-\langle\alpha, g\rangle\right\|_{\Lambda_{2}(\mu)}$ converges to 0 . Thus by the isomorphism, the sampling theorem (12) is verified with $a_{g}(h)=\hat{F}_{g}(h)$.

The character group of $\hat{G} / A$ is isomorphic to $H$, under the correspondence $\langle h, T(\alpha)\rangle=\langle\alpha, h\rangle$, so the Fourier transform of $F_{g}$ is $F_{g}(h)=$ $\int_{\hat{G} / A}\langle\alpha, h\rangle F_{g}(T(\alpha)) M_{\hat{G} / A}(d T(\alpha))$ where $M_{\hat{G} / A}$ is the Haar measure on $\hat{G} / A$. Now the Haar measures on $\hat{G}, A$ and $\hat{G} / A$ are related by the equation

$$
M_{\hat{G}}(W)=\int_{\hat{G} / A} \int_{A} \chi_{W}(\alpha+\gamma) M_{A}(d \gamma) M_{\hat{G} / A}(d T(\alpha))
$$

where $\chi_{W}$ is the indicator of $W$. The inner integral is a function of $T(\alpha)$ since $\int \chi_{\Delta}(\alpha+\gamma) M_{A}(d \gamma)$ is unchanged if $\alpha$ is replaced by $\alpha+\gamma$ for $\gamma \in A$. Now

$$
\int_{A} \chi_{W}(\alpha+\gamma) M_{A}(d \gamma)= \begin{cases}M_{A}\left(\left\{\gamma_{0}\right\}\right) & \text { if } \alpha+\gamma_{0} \in W \text { for some } \gamma_{0} \in A, \\ 0 & \text { otherwise }\end{cases}
$$

since the translates of $W$ are disjoint. Thus $\int_{A} \chi_{W}(\alpha+\gamma) M_{A}(d \alpha)=M_{A}(\{e\})$ for $T(\alpha) \in T(W)$ and zero otherwise and so $M_{\hat{G}}(W)=M_{A}(\{e\})$. $M_{\hat{G} / A}(T(W))$ and thus is finite.

If $A$ is discrete, then $\int|\psi(\alpha+\gamma)\langle\alpha+\gamma, g-h\rangle| M_{A}(d \gamma)=\Sigma_{\gamma}|\psi(\alpha+\gamma)|<$ $\infty$ so $\psi(\alpha+\gamma)\langle\alpha+\gamma, g-h\rangle \in L_{1}(A)$ and $F_{g}(T(\alpha))=\int_{A} \psi(\alpha+\gamma)\langle\alpha+$ $\gamma, g>M_{A}(d \gamma)$. Then $\hat{F}_{g}(h)$ equals 


$$
\begin{aligned}
\int_{\hat{G} / A} F_{g}(T(\alpha)) \overline{\langle h, T(\alpha)\rangle} M_{\hat{G} / A}(d T(\alpha)) \\
\quad=\int_{\hat{G} / A} \int_{A} \psi(\alpha+\gamma)\langle\alpha+\gamma, g\rangle M_{A}(d \alpha) \overline{\langle\alpha, h\rangle} M_{\hat{G} / A}(d(T \alpha)) \\
=\int_{\hat{G} / A} \int_{A} \psi(\alpha+\gamma)\langle\alpha+\gamma, g-h\rangle M_{A}(d \alpha) M_{\hat{G} / A}(d T(\alpha)) \\
=\int_{\hat{G}}\langle\alpha, g-h\rangle \psi(\alpha) M_{\hat{G}}(d \alpha)
\end{aligned}
$$

and so $a_{g}(h)=\hat{\psi}(g-h)$.

If $A$ is not discrete then

$$
\begin{aligned}
\hat{F}_{g}(h) & =\int_{\hat{G} / A} F_{g}(T(\alpha)) \overline{\langle\alpha, h\rangle} M_{\hat{G} / A}(d T(\alpha)) \\
& =\int_{T(W)} F_{g}(T(\alpha)) \overline{\langle\alpha, h\rangle} M_{\hat{G} / A}(d T(\alpha))
\end{aligned}
$$

since $F_{g}$ is zero off $T(W)$. For the general case, consider the $\sigma$-field of Borel subsets of $W$. If $U$ is an open subset of $W$, then $T(U)$ is an open subset of $\hat{G} / A$. Since $T$ is a 1-1 correspondence between $W$ and $T(W)$, the set function $\nu$ defined by $\nu(U)=M_{\hat{G} / A}(T(U))$ is a countably additive finite set function on the open sets of $W$ which may be extended to a finite measure on the Borel sets of $W$. Note also that for any Borel subset $\Delta$ of $T(W), \nu T^{-1}(\Delta)=$ $M_{\hat{G} / A}(\Delta)$. Thus by the change of variable formula

$$
\begin{aligned}
\hat{F}_{g}(h) & =\int_{T(W)} F_{g}(T(\alpha)) \overline{\langle h, T(\alpha)\rangle} M_{\hat{G} / A}(d T(\alpha)) \\
& =\int_{W} F_{g}(\alpha) \overline{\langle\alpha, h\rangle} \nu(d \alpha)=\int_{W} \psi(\alpha)\langle\alpha, g-h\rangle \nu(d \alpha) .
\end{aligned}
$$

If $A$ is discrete then $M_{\hat{G}}(U)$ is proportional to $\nu(U)$ for all Borel subsets of $W$.

Note. The restriction on $H$ (that it be an infinite finitely generated discrete group) is not necessary for Theorem 9 to hold. Any discrete subgroup $H$ whose character group $\hat{H}$ possesses a faithful finite dimensional representation will serve, provided slight modifications in the statement of Theorem 9 are made.

\section{REFERENCES}

1. M. Zakai, Bandlimited functions and the sampling theorem, Information and Control 8 (1965), 143-158. MR 30 \#4607.

2. Z. A. Piranashvili, On the problem of interpolation of stochastic processes, Theor. Probability Appl. 12 (1967), 647-657.

3. A. J. Lee, On bandlimited stochastic processes, SIAM J. Appl. Math. 30 (1976), 269-277. 
4. A. J. Lee, Characterization of bandlimited functions and processes, Information and Control 31 (1976), 258-271.

5. S. P. Lloyd, $A$ sampling theorem for stationary (wide sense) stochastic processes, Trans. Amer. Math. Soc. 92 (1959), 1-12. MR 21 \#6026.

6. M. M. Rao, Inference in stochastic processes. III, Z. Wahrscheinlichkeitstheorie und Verw. Gebiete 8 (1967), 49-72. MR 35 \# 7542.

7. S. Cambanis and E. Masry, On the representation of weakly continuous stochastic processes, Information Sci. 3 (1971), 289-290. MR 46 \#4607.

8. E. Parzen, An approach to time series analysis, Ann. Math. Statist. 32 (1961), 951-989. MR 26 \#874.

9. A. H. Zemanian, Distribution theory and transform analysis, McGraw-Hill, New York, 1965. MR 31 \# 1556.

10. F. Trèves, Topological vector spaces, distributions and kernels, Academic Press, New York, 1967. MR 37 \# 726.

11. W. Rudin, Fourier analysis on groups, Wiley, New York, 1962. MR 27 \#2808.

12. S. Cambanis and B. Liu, On harmonizable stochastic processes, Information and Control 17 (1970), 183-202. MR 43 \#8113.

13. E. Hewitt and K. A. Ross, Abstract harmonic analysis, Vol. 1, Springer-Verlag, Berlin and New York, 1963. MR 28 \# 158.

14. R. A. Mayer, Summation of Fourier series on compact groups, Amer. J. Math. 89 (1967), 661-692. MR 36 \# 1578.

Department of Mathematics, University of Auckland, Private Bag, Auckiand, New ZeALAND 